Reprod. Nutr. Develop., 1988, 28 (3 B), 753-756

\title{
Isoformes de la myosine synthétisées au cours de la régénération de muscles squelettiques à contraction rapide, en présence du nerf moteur et après dénervation. Etude chez le rat et la souris adultes
}

\author{
Anne d'ALBIS $\left({ }^{1}\right)$, R. COUTEAUX $\left({ }^{2}\right)$, J. C. MIRA $\left({ }^{2}\right)\left({ }^{3}\right)$, Chantal JANMOT $\left({ }^{1}\right)$, \\ Agnès ROULET $\left({ }^{1}\right)$ \\ ( $\left.{ }^{1}\right)$ Laboratoire de Biologie Physico-chimique, UA C.N.R.S., n $n^{\circ} 1131$, \\ Université Paris-Sud, 91405 Orsay. \\ ( $\left.{ }^{2}\right)$ Laboratoire de Cytologie, UA C.N.R.S. $n^{\circ} 1199$, \\ Université Pierre et Marie Curie, 75006 Paris. \\ $\left({ }^{3}\right)$ Laboratoire de Neurobiologie, Université René Descartes \\ 45, rue des Saints-Pères, 75006 Paris.
}

Summary. Isomyosins synthetized during regeneration of innervated and denervated fast contracting skeletal muscles. Study in adult rats and mice.

Adult rat and mouse fast contracting skeletal muscles were injured by a cardiotoxin. New myosins of embryonic, neonatal and adult types appeared 4 and 5 days after the treatment in both innervated and denervated muscles. Although their structure remained altered, innervated - but not denervated -- muscles rapidly recovered a normal isomyosin pattern.

II est généralement admis qu'un muscle lésé régénère à partir de cellules satellites et que la nouvelle myogenèse « récapitule » la myogenèse du développement (revue dans Plaghki, 1985 et Swinghedauw, 1986). L'environnement neuroendocrinien étant différent, on peut s'attendre cependant à certaines différences entre les deux myogenèses. Nous avons donc étudié morphologiquement et biochimiquement la régénération de muscles à contraction rapide de rats et de souris adultes et d'autre part examiné l'effet de la dénervation sur cette régénération.

Les muscles (sternomastoïdiens, lombricaux et intercostaux externes) sont traités par une cardiotoxine extraite du venin de Naja mossambica mossambica ( $P$. Bougis, Laboratoire de Biochimie, Marseille) et prélevés à intervalles réguliers entre 24 h et 2 mois après le traitement. Des coupes transversales sont examinées par microscopie optique et la distribution des récepteurs cholinergiques est étudiée par microscopie de fluorescence à l'aide d'alpha-bungarotoxine marquée par de l'isothiocyanate de tétra-méthyl-rhodamine. Les différents types de myosines synthétisées sont analysés par électrophorèse en conditions non dissociantes (myosine entière) et dissociantes (chaînes légères). 
Régénération en présence du nerf.

De nouvelles myosines sont détectables à partir des $4^{\mathrm{e}}$ et $5^{\mathrm{e}}$ jours après le traitement des muscles par la cardiotoxine. Des myosines de type embryonnaire, néonatal, adulte intermédiaire et rapide sont synthétisées. La forme embryonnaire n'est plus observée après 6 jours et les formes néonatales disparaissent rapidement après 8 jours. A partir de deux semaines après le traitement, le muscle sternomastoïdien présente une distribution normale de myosines adultes de type intermédiaire et rapide (fig. 1 et 2 ).

a

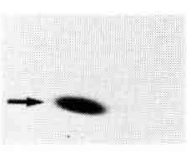

b

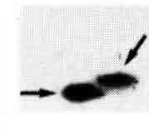

c

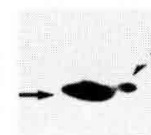

d

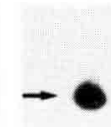

e

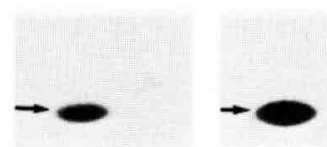

FIG. 1. - Chaîne légère de type 1 de myosine de sternomastoidien de rat adulte. Analyse sur gel de polyacrylamide $15 \%$ en présence de SDS après électrofocalisation.

a: Témoin ; b-f: Muscles traités par la cardiotoxine, respectivement $4,5,6,8$ et 14 jours après le traitement.

$\checkmark$ chaîne légère embryonnaire ; $\rightarrow$ chaîne légère néonatale, intermédiaire ou adulte.
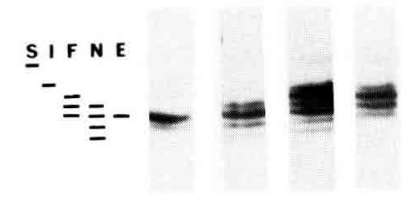

a b c d

A
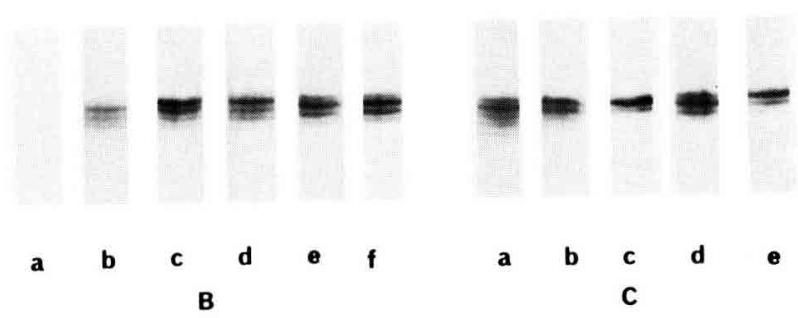

FIG. 2. - Isomyosines natives de sternomastö̈dien de rat. Analyse en conditions non-dissociantes. A : Témoins. a : Embryon de 18 jours. $b$ : Rat de 3 jours. $c$ : Rat de 12 jours. d : Adulte.

$B$ : Muscles innervés lors de la régénération. a- $f$ : respectivement $3,4,5,8,14$ et 62 jours après le traitement par la cardiotoxine.

$C$ : Muscles dénervés lors de la régénération. a-e : respectivement $8,14,21,42$ et 62 jours après le traitement par la cardiotoxine.

Isomyosines: $S$, lente ; $I$, intermédiaire ; $F$, rapide ; $N$, néonatale ; $E$, embryonnaire.

L'aspect structural du muscle régénéré n'est cependant pas totalement conforme à celui d'un muscle témoin ; les fibres sont moins jointives, leur diamètre est plus variable, de nombreux noyaux centraux persistent (fig. 3a et b), et la jonction neuromusculaire présente une terminaison en grappe au lieu de la terminaison normale en plaque comme le montre la répartition des récepteurs cholinergiques (fig. $3 c$ et $d$ ). 

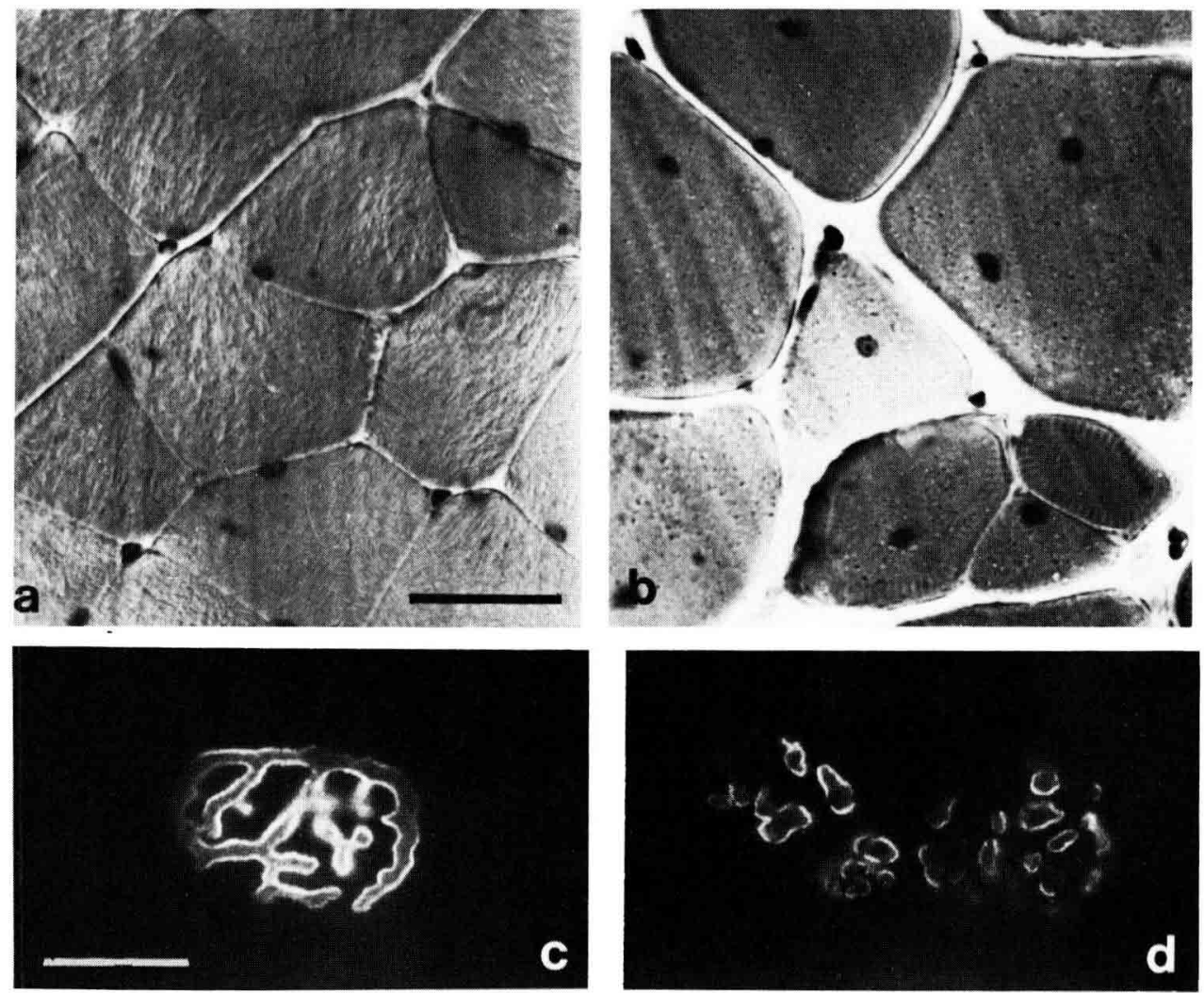

FIG. 3. - a et b. - Sternomastoïdien de souris. Coloration des noyaux par le carmalum. Barre : $30 \mu \mathrm{m}$. $a$ : Témoin ; $b: 9$ mois après le traitement par la cardiotoxine.

$c$ et $d$. - Intercostal externe de souris. Distribution des récepteurs cholinergiques à la jonction neuromusculaire. Barre : $20 \mu \mathrm{m}$. $c$ : Témoin ; $d: 51$ jours après le traitement par la cardiotoxine.

\section{Régénération en absence du nerf.}

Les muscles préalablement dénervés par résection du nerf moteur synthétisent au début de la régénération les mêmes types de myosine que les muscles non dénervés. Par la suite, les myosines de type néonatal persistent plus longtemps et les muscles ne recouvrent pas un diagramme normal d'isomyosines; la forme intermédiaire adulte est généralement favorisée au détriment des formes rapides (fig. 2).

Comme au cours de la myogenèse normale (Whalen et al., 1981), différents types de myosine sont donc synthétisés au cours de la régénération de muscles adultes. Cependant, nous avons observé que l'apparition des myosines de type adulte se fait plus rapidement dans la régénération; cette accélération peut 
s'expliquer par un environnement et des facteurs de régulation différents chez l'adulte et peut-être également par la non-équivalence entre les cellules satellites et les myoblastes embryonnaires (Chevallier et al., 1987). L'innervation et très certainement l'activité contractile du muscle (Lömo et al., 1974) apparaissent d'autre part essentielles pour le rétablissement d'un contenu normal en isomyosines.

$13^{e}$ Réunion du groupe Développement I.N.R.A., Cap d'Agde, 25-27 mai 1987.

\section{Références}

CHEVAllier A., PAUTOU M. P., HARRIS A. J., KIENY M., 1987. On the non-equivalence of skeletal muscle satellite cells and embryonic myoblasts. Arch. Anat. micr. Morph. exp., 75. 161-166.

LÖMO T., WESTGAARD R. H., DAHL H. A., 1974. Contractile properties of muscle : control by pattern of muscle activity in the rat. Proc. roy. Soc. Lond. B. Biol. Sci., 187, 99-103.

PLAGHKI L., 1985. Régénération et myogenèse du muscle strié. J. Physio/. (Paris), 80, $51-110$. SWINGHEDAUW B., 1986. Developmental and functional adaptation of contractile proteins in cardiac and skeletal muscles. Physiol. Rev., 66, 710-771.

WHALEN R. G., SELL S. M., BUTLER-BROWNE G. S., SCHWARTZ K., BOUVERET P., PINSETHARSTRÖM I., 1981. Three myosin heavy chain isozymes appear sequentially in rat muscle development. Nature (London), 292, 805-809. 